\title{
Rota changes in acute and general internal medicine: delivering improved patient care and junior doctor training at the front and back door - acute medical unit placement blocks the way forward for Midland Met!
}

\author{
Authors: Richard Buka, Donna Best and Sarbjit Clare
}

\section{Aims}

Introduction of a rota to provide consistency of care for patients and improved training for junior doctors.

\section{Methods \\ Initial meetings were held with stakeholders from medical staffing, consultant and junior doctors. Following consultation, a prototype rota was agreed for foundation doctors and 'senior house officer' grades whereby doctors rotate into acute medicine for 5 or 6 -week blocks once every 4 months. Within this block is two sets of nights, three to four weekends, late shifts and some normal working days. The remainder of the 4-month block would be spent back in their specialty rotation mostly working weekdays $9 \mathrm{am}-5 \mathrm{pm}$. Two $9 \mathrm{am}-$ $5 \mathrm{pm}$ ward cover doctors were added to support the weekends. \\ The rota changes occurred in line with the August changeover of junior doctors in 2017. An electronic survey was sent out after junior doctors had completed their first 6-week rotation in the acute medical unit (AMU). Numbers of patients handed over were assessed by looking at the referrals book.}

\section{Results}

Previously in a 24 -hour period there were 78 junior doctor hours dedicated to seeing new acute medical patients during the week. With the new rota, there are 100 junior doctor hours in 24 hours during the week. Hours for new patient care at the weekend remain the same but an extra 16 hours in 24 hours has been added for ward cover.

The mean number of patients handed over at the end of shifts after the institution of the new rota was significantly lower (before, 4.2; after, 7.8; $p=0.005$ ). There was significant difference in weekend handover numbers. There was no significant difference in total number of patients seen.

Authors: Sandwell and West Birmingham Hospitals NHS Trust, Birmingham, UK
Responses to the junior doctor survey were received from 18 of 28 (64\%) participants: 78\% enjoyed working on the new rota; $83 \%$ agreed that they had adequate opportunities to obtain workplace-based assessments compared with $55 \%$ beforehand. Mean score for work-life balance was 57/100. Mean score for quality of senior support was $90 / 100$ and mean overall rating for the placement was $82 / 100$.

In addition the AMU has a dedicated clinical skills lab and a focused induction as well as a practical skills teaching programme, which has empowered the AMU placement junior doctors, who previously would not have had this opportunity to utilise and have exposure to such procedures.

\section{Conclusion}

Previously, junior doctor staffing did not match emergency admissions and weekend ward cover was very demanding resulting in increased patients handed over at nights and thus an out of proportion workload. Our data show that these rota changes successfully support front-door delivery of acute medical care with fewer patients handed over at the end of shifts thus waiting times are shorter.

Feedback from junior doctors was largely positive and the rotation system has allowed for formal teaching programmes within acute medicine.

The main negative was that the wards were sometimes shortstaffed during the week. We have now taken steps to mitigate this effect with junior doctors returning to their base ward on normal working days. Long term planning is underway to support the wards with allied health professionals. We will now extend these rota changes to our sister hospital, Sandwell, with the view to progression to Midland Met which is our super acute hospital due to open in April 2019.

\section{Conflict of interest statement}

None declared. 